\title{
DIFFERENTIAL OPERATORS FOR HARMONIC WEAK MAASS FORMS AND THE VANISHING OF HECKE EIGENVALUES
}

\author{
JAN H. BRUINIER, KEN ONO AND ROBERT C. RHOADES
}

\begin{abstract}
For integers $k \geq 2$, we study two differential operators on harmonic weak Maass forms of weight $2-k$. The operator $\xi_{2-k}$ (resp. $D^{k-1}$ ) defines a map to the space of weight $k$ cusp forms (resp. weakly holomorphic modular forms). We leverage these operators to study coefficients of harmonic weak Maass forms. Although generic harmonic weak Maass forms are expected to have transcendental coefficients, we show that those forms which are "dual" under $\xi_{2-k}$ to newforms with vanishing Hecke eigenvalues (such as $\mathrm{CM}$ forms) have algebraic coefficients. Using regularized inner products, we also characterize the image of $D^{k-1}$.
\end{abstract}

\section{Introduction and Statement of Results}

Let $M_{k}^{!}\left(\Gamma_{0}(N), \chi\right)$ denote the space of integer weight $k$ weakly holomorphic modular forms on $\Gamma_{0}(N)$ with Nebentypus $\chi$. Recall that a weakly holomorphic modular form is any meromorphic modular form whose poles (if any) are supported at cusps. Weakly holomorphic modular forms naturally sit in spaces of harmonic weak Maass forms (see Section 2 for definitions), more general automorphic forms which have been a source of recent interest due to their connection to Ramanujan's mock theta functions, Borcherds products, derivatives of modular $L$-functions, and traces of singular moduli (see [2, 3, 4, [5, 6, 7, [8, 20, 21]).

In view of these applications, it is natural to investigate the arithmeticity of the Fourier coefficients of such Maass forms, and to also investigate their nontrivial interplay with holomorphic and weakly holomorphic modular forms. In the works above, one such nontrivial relationship (see Prop. 3.2 of [7]), involving the differential operator

$$
\xi_{w}:=2 i y^{w} \cdot \frac{\bar{\partial}}{\partial \bar{z}},
$$

plays a central role. It is the fact that

$$
\xi_{2-k}: H_{2-k}\left(\Gamma_{0}(N), \chi\right) \longrightarrow S_{k}\left(\Gamma_{0}(N), \bar{\chi}\right) .
$$

Here $H_{w}\left(\Gamma_{0}(N), \chi\right)$ denotes the space of weight $w$ harmonic weak Maass forms on $\Gamma_{0}(N)$ with Nebentypus $\chi$, and $S_{w}\left(\Gamma_{0}(N), \chi\right)$ denotes the subspace of cusp forms. It is not

The second author thanks the generous support of the National Science Foundation, and the Manasse family. The third author is grateful for the support of a National Physical Sciences Consortium Graduate Fellowship, and a National Science Foundation Graduate Fellowship. 
difficult to make this more precise using Fourier expansions. In particular, every weight $2-k$ harmonic weak Maass form $f(z)$ has a Fourier expansion of the form

$$
f(z)=\sum_{n \gg-\infty} c_{f}^{+}(n) q^{n}+\sum_{n<0} c_{f}^{-}(n) \Gamma(k-1,4 \pi|n| y) q^{n}
$$

where $\Gamma(a, x)$ is the incomplete Gamma-function, $z=x+i y \in \mathbb{H}$, with $x, y \in \mathbb{R}$, and $q:=e^{2 \pi i z}$. A straightforward calculation shows that $\xi_{2-k}(f)$ has the Fourier expansion

$$
\xi_{2-k}(f)=-(4 \pi)^{k-1} \sum_{n=1}^{\infty} \overline{c_{f}^{-}(-n)} n^{k-1} q^{n} .
$$

As (1.2) reveals, $f(z)$ naturally decomposes into two summands

$$
\begin{gathered}
f^{+}(z):=\sum_{n \gg-\infty} c_{f}^{+}(n) q^{n}, \\
f^{-}(z):=\sum_{n<0} c_{f}^{-}(n) \Gamma(k-1,4 \pi|n| y) q^{n} .
\end{gathered}
$$

Therefore, $\xi_{2-k}(f)$ is given simply in terms of $f^{-}(z)$, the non-holomorphic part of $f$.

Here we show that $f^{+}(z)$, the holomorphic part of $f$, is also intimately related to weakly holomorphic modular forms. We require the differential operator

$$
D:=\frac{1}{2 \pi i} \cdot \frac{d}{d z} \text {. }
$$

Theorem 1.1. If $2 \leq k \in \mathbb{Z}$ and $f \in H_{2-k}\left(\Gamma_{0}(N), \chi\right)$, then

$$
D^{k-1}(f) \in M_{k}^{!}\left(\Gamma_{0}(N), \chi\right) .
$$

Moreover, assuming the notation in (1.2), we have

$$
D^{k-1} f=D^{k-1} f^{+}=\sum_{n \gg-\infty} c_{f}^{+}(n) n^{k-1} q^{n} .
$$

Remark 1. Theorem [1.1 is related to classical results on weakly holomorphic modular forms and Eichler integrals. Theorem 1.1 is a generalization of the classical result on weakly holomorphic modular forms to the context of harmonic weak Maass forms.

Theorem 1.1 implies that the coefficients $c_{f}^{+}(n)$, for non-zero $n$, are obtained by dividing the $n$th coefficient of some fixed weakly holomorphic modular form by $n^{k-1}$. Therefore we are compelled to determine the image of the map

$$
D^{k-1}: H_{2-k}\left(\Gamma_{0}(N), \chi\right) \longrightarrow M_{k}^{!}\left(\Gamma_{0}(N), \chi\right) .
$$

It is not difficult to see that this map is not generally surjective. Our next result determines the image of $D^{k-1}$ in terms of "regularized" inner products (see Section 4).

Theorem 1.2. If $2 \leq k \in \mathbb{Z}$, then the image of the map

$$
D^{k-1}: H_{2-k}\left(\Gamma_{0}(N), \chi\right) \longrightarrow M_{k}^{!}\left(\Gamma_{0}(N), \chi\right)
$$

consists of those $h \in M_{k}^{!}\left(\Gamma_{0}(N), \chi\right)$ which are orthogonal to cusp forms (see Section 4) which also have constant term 0 at all cusps of $\Gamma_{0}(N)$. 
Although these results for $D^{k-1}$ suggest that one has complete information concerning the Fourier coefficients of $f^{+}$, it turns out that some of the most basic questions remain open. Here we consider algebraicity. Despite the fact that we have a fairly complete theory of algebraicity for forms in $M_{k}^{!}\left(\Gamma_{0}(N), \chi\right)$, thanks to the $q$-expansion principle, the theory of Eisenstein series and newforms, this question remains open for harmonic weak Maass forms. In view of the theory of newforms, it is natural to restrict our attention to those $f \in H_{2-k}\left(\Gamma_{0}(N), \chi\right)$ for which $\xi_{2-k}(f) \in S_{k}\left(\Gamma_{0}(N), \bar{\chi}\right)$ is a Hecke eigenform. For reasons which will become apparent, we shall concentrate on those forms for which

$$
\xi_{2-k}(f)=\frac{g}{\|g\|^{2}}
$$

where $g$ is a normalized newform and $\|g\|$ denotes its usual Petersson norm.

To illustrate the nature of this problem, we consider two examples of Maass-Poincaré series which are not weakly holomorphic modular forms. The Maass-Poincaré series (see Section [6] $f:=Q(-1,12,1 ; z) \in H_{-10}\left(\mathrm{SL}_{2}(\mathbb{Z})\right.$ ) (note. If a Nebentypus character is not indicated, then it is assumed to be trivial) satisfies (1.7) for $g=\Delta(z)$, the unique normalized weight 12 cusp form on the full modular group. The first few coefficients of its holomorphic part are

$Q^{+}(-1,12,1 ; z) \sim q^{-1}-0.04629-1842.89472 q-23274.07545 q^{2}-225028.75877 q^{3}-\cdots$.

There is little reason to believe that these coefficients are rational or algebraic. On the other hand, we shall prove that the Maass-Poincaré series $Q(-1,4,9 ; z) \in H_{-2}\left(\Gamma_{0}(9)\right)$ has the property that $Q^{+}(-1,4,9 ; z)$ has rational coefficients. Its first few terms are

$$
Q^{+}(-1,4,9 ; z)=q^{-1}-\frac{1}{4} q^{2}+\frac{49}{125} q^{5}-\frac{48}{512} q^{8}-\frac{771}{1331} q^{11}+\cdots
$$

and $f:=Q(-1,4,9 ; z)$ satisfies (1.7) for the unique normalized newform in $S_{4}\left(\Gamma_{0}(9)\right)$.

Our next result explains the distinction between these two cases. To make this precise, let $g \in S_{k}\left(\Gamma_{0}(N), \bar{\chi}\right)$ be a normalized newform, and let $F_{g}$ be the number field obtained by adjoining the coefficients of $g$ to $\mathbb{Q}$. We say that a harmonic weak Maass form $f \in H_{2-k}\left(\Gamma_{0}(N), \chi\right)$ is good for $g$ if it satisfies the following properties (Section 2 for definitions):

(i) The principal part of $f$ at the cusp $\infty$ belongs to $F_{g}\left[q^{-1}\right]$.

(ii) The principal parts of $f$ at the other cusps of $\Gamma_{0}(N)$ are constant.

(iii) We have that $\xi_{2-k}(f)=\|g\|^{-2} g$.

Remark 2. For every such $g$, Proposition 5.1 will show that there is an $f$ which is good for $g$. Moreover, such an $f$ is unique up to a weakly holomorphic form in $M_{2-k}^{!}\left(\Gamma_{0}(N), \chi\right)$ with coefficients in $F_{g}$. Such $f$ can be constructed explicitly using Poincaré series (for example, see Section 6 for even $k \geq 2$ and trivial Nebentypus).

Theorem 1.3. Let $g \in S_{k}\left(\Gamma_{0}(N), \bar{\chi}\right)$ be a normalized newform with complex multiplication. If $f \in H_{2-k}\left(\Gamma_{0}(N), \chi\right)$ is good for $g$, then all coefficients of $f^{+}$are in $F_{g}\left(\zeta_{M}\right)$, where $\zeta_{M}:=e^{2 \pi i / M}$, and $M=N D$ where $D$ is the discriminant of the field of complex multiplication. 
Remark 3 .

i) The rationality of $Q^{+}(-1,4,9 ; z)$ in (1.8) is an example of Theorem 1.3. In this case $Q(-1,4,9 ; z)$ is good for the unique CM newform in $S_{4}\left(\Gamma_{0}(9)\right)$. We shall discuss this example in detail in the last section.

ii) The field $F_{g}$ in Theorem 1.3 is explicit (see the discussion in Section 5).

iii) Suppose that $g \in S_{k}\left(\Gamma_{0}(N), \bar{\chi}\right)$ is a normalized newform. If $f \in H_{2-k}\left(\Gamma_{0}(N), \chi\right)$ is good for $g$, then the proof of Theorem 1.3 implies that all of the coefficients of $f^{+}$belong to $F_{g}\left(c_{f}^{+}(1)\right)$. It would be interesting to describe this field in terms of intrinsic invariants associated to $g$.

iv) It is interesting to compare Theorem 1.3, which concerns integer weights $2-k$, with the results in [8] which pertain to weight $1 / 2$ harmonic weak Maass forms. The first two authors proved that if $g$ is a newform of weight $3 / 2$ which is orthogonal to all elementary theta series, and if $f$ is defined analogously as above, then

$$
\begin{array}{r}
\#\left\{n \in \mathbb{N}: c_{f}^{+}(n) \text { transcendental }\right\}=+\infty, \\
\#\left\{n \in \mathbb{N}: c_{f}^{+}(n) \text { algebraic }\right\}=+\infty .
\end{array}
$$

In fact, estimates are obtained for these quantities. These results are related to the vanishing of derivatives of quadratic twists of weight 2 modular $L$-functions at $s=1$.

v) It would be interesting to find an explicit construction of good harmonic weak Maass forms for CM newforms. Perhaps there is a construction which is analogous to the case of the mock theta functions [2, 3, [5, 20, 21].

vi) In the examples we know, it turns out that the coefficients of $f^{+}$are actually contained in $F_{g}$. It seems natural to ask whether this is true in general.

The proof of Theorem 1.3 relies on the fact that some Hecke eigenvalues of $g$ vanish. A simple generalization of the proof of Theorem 1.3 can be used to detect the vanishing of the Fourier coefficients of a newform.

Theorem 1.4. Suppose that $g=\sum_{n=1}^{\infty} c_{g}(n) q^{n} \in S_{k}\left(\Gamma_{0}(N), \bar{\chi}\right)$ is a normalized newform, and suppose that $f \in H_{2-k}\left(\Gamma_{0}(N), \chi\right)$ is good for $g$. If $p \nmid N$ is a prime for which $c_{g}(p)=0$, then $c_{f}^{+}(n)$ is algebraic when $\operatorname{ord}_{p}(n)$ is odd.

Remark 4. The proof of Theorem 1.4 shows that the coefficients of $f^{+}$are in an explicit abelian extension of $F_{g}$ when $c_{g}(p)=0$. It seems possible that the coefficients of $f^{+}$are always in $F_{g}$ when there are any vanishing Hecke eigenvalues. As the next example will show, this is the case when $N=1$.

Example. Here we consider Lehmer's Conjecture on the nonvanishing of Ramanujan's $\tau$-function, where

$$
\Delta(z)=\sum_{n=1}^{\infty} \tau(n) q^{n} .
$$

This example generalizes easily to all level 1 Hecke eigenforms. 
Although Theorem 1.4 relates Lehmer's Conjecture to the alleged transcendence of the coefficients, say $a_{\Delta}(n)$, of $Q^{+}(-1,12,1 ; z)$, it turns out that much more is true. Lehmer's Conjecture is implied by the mere irrationality of any these coefficients.

We make use of explicit formulas. Using the classical Eisenstein series $E_{4}$ and $E_{6}$ and the classical $j$-function $j(z)$, we define polynomials $J_{m}(x)$ by

$$
\sum_{m=0}^{\infty} J_{m}(x) q^{m}:=\frac{E_{4}(z)^{2} E_{6}(z)}{\Delta(z)} \cdot \frac{1}{j(z)-x}=1+(x-744) q+\cdots .
$$

For each $m$ we then let $j_{m}(z)=J_{m}(j(z))$. If $p$ is prime, then define the modular functions

$$
\begin{gathered}
A_{p}(z):=\frac{24}{B_{12}}\left(1+p^{11}\right)+j_{p}(z)-264 \sum_{m=1}^{p} \sigma_{9}(m) j_{p-m}(z), \\
B_{p}(z):=-\tau(p)\left(-264+\frac{24}{B_{12}}+j_{1}(z)\right) .
\end{gathered}
$$

Here $B_{12}=-691 / 2730$ is the 12 th Bernoulli number, and $\sigma_{9}(n):=\sum_{d \mid n} d^{9}$. Using the principal part of $Q(-1,12,1 ; z)$ combined with the fact that $\Delta(z)$ is an eigenform of the Hecke algebra, one can show (for example, see [14]), for primes $p$, that

$$
\sum_{n=-p}^{\infty}\left(p^{11} a_{\Delta}(p n)-\tau(p) a_{\Delta}(n)+a_{\Delta}(n / p)\right) q^{n}=\frac{A_{p}(z)+B_{p}(z)}{E_{4}(z) E_{6}(z)} .
$$

These weight -10 modular forms have integer coefficients. Now suppose that $\tau(p)=0$ for a prime $p$. Then $a_{\Delta}(n p)$ is rational for every $n$ coprime to $p$. Under this assumption, the proof of Theorem 1.3 then implies that $a_{\Delta}(n)$ is rational when $\operatorname{ord}_{p}(n)$ is odd.

Due to Theorems 1.3 and 1.4, it is natural to consider the arithmetic properties of harmonic weak Maass forms. For brevity, we will be content with the following result for certain forms with prime power level and trivial Nebentypus.

Theorem 1.5. Suppose that $p$ is prime, and that $f(z) \in H_{2-k}\left(\Gamma_{0}\left(p^{t}\right)\right)$ is good for a newform $g \in S_{k}\left(\Gamma_{0}\left(p^{t}\right)\right)$ with complex multiplication. If we let

$$
a:=\min \left\{d \geq 0: c_{f}^{+}\left(p^{d} n\right)=0 \text { for all } n<0\right\},
$$

then the following are true:

1) The formal q-series

$$
f^{*}:=\sum_{n=0}^{\infty} c_{f}^{+}\left(p^{a} n\right) n^{k-1} q^{n}
$$

is a p-adic modular form on $\mathrm{SL}_{2}(\mathbb{Z})$ of weight $k$.

2) For every positive integer $b$, we have that

$$
\lim _{X \rightarrow+\infty} \frac{\#\left\{n \leq X: c_{f}^{+}\left(p^{a} n\right) n^{k-1} \not \equiv 0 \quad\left(\bmod p^{b}\right)\right\}}{X}=0 .
$$


3) If $p \leq 7$, or $p \geq 11$ and $k \equiv 4,6,8,10,14(\bmod p-1)$, then as $p$-adic numbers we have

$$
\lim _{n \rightarrow+\infty} c_{f}^{+}\left(p^{a+n}\right) p^{n(k-1)}=0
$$

Remark 5. Theorem 1.5 (2) says that "almost every" $c_{f}^{+}\left(p^{a} n\right)$ is a multiple of $p^{b}$. Theorem 1.5 (3) is not a trivial statement since the coefficients $c_{f}^{+}\left(p^{a+n}\right)$ tend to have unbounded denominators involving increasing powers of $p$.

In Section 2 we recall definitions and facts about harmonic weak Maass forms and their behavior under certain differential operators. In Section 3 we prove Theorem 1.1. In Section 4 we recall facts about the regularized inner product, which generalizes Petersson's inner product, and we prove Theorem 1.2. In Section 5 we prove Theorems 1.3, 1.4 and 1.5, and in Section 6 we illustrate Theorems 1.1 and 1.2 using Poincaré series. In the last section, we examine example (1.8) in the context of all of the results above.

\section{ACKNOWLEDGEMENTS}

The authors thank the referee for several helpful suggestions and corrections.

\section{HARMONIC WEAK MAASS FORMS}

Here we recall definitions and facts about harmonic weak Maass forms. Throughout, let $z=x+i y \in \mathbb{H}$, the upper-half of the complex plane, with $x, y \in \mathbb{R}$. Also, throughout suppose that $k \in \mathbb{N}$. We define the weight $k$ hyperbolic Laplacian by

$$
\Delta_{k}:=-y^{2}\left(\frac{\partial^{2}}{\partial x^{2}}+\frac{\partial^{2}}{\partial y^{2}}\right)+i k y\left(\frac{\partial}{\partial x}+i \frac{\partial}{\partial y}\right) .
$$

Suppose that $\chi$ is a Dirichlet character modulo $N$. Then a harmonic weak Maass form of weight $k$ on $\Gamma_{0}(N)$ with Nebentypus $\chi$ is any smooth function on $\mathbb{H}$ satisfying:

(i) $f\left(\frac{a z+b}{c z+d}\right)=\chi(d)(c z+d)^{k} f(z)$ for all $\left(\begin{array}{ll}a & b \\ c & d\end{array}\right) \in \Gamma_{0}(N)$;

(ii) $\Delta_{k} f=0$;

(iii) There is a polynomial $P_{f}=\sum_{n \leq 0} c_{f}^{+}(n) q^{n} \in \mathbb{C}\left[q^{-1}\right]$ such that $f(z)-P_{f}(z)=$ $O\left(e^{-\varepsilon y}\right)$ as $y \rightarrow \infty$ for some $\varepsilon>0$. Analogous conditions are required at all cusps.

The polynomial $P_{f} \in \mathbb{C}\left[q^{-1}\right]$ is called the principal part of $f$ at the corresponding cusp. We denote the vector space of these harmonic weak Maass forms by $H_{k}\left(\Gamma_{0}(N), \chi\right)$

Remark 6. Note that our definition slightly differs from the one of [7], since we assume that the singularities of $f$ at the cusps are supported on the holomorphic parts of the corresponding Fourier expansions. This space is denoted by $H_{k}^{+}$in [7]. 
Recall the Maass raising and lowering operators (see [7, 9]) $R_{k}$ and $L_{k}$ on functions $f: \mathbb{H} \rightarrow \mathbb{C}$ which are defined by

$$
\begin{aligned}
& R_{k}=2 i \frac{\partial}{\partial z}+k y^{-1}=i\left(\frac{\partial}{\partial x}-i \frac{\partial}{\partial y}\right)+k y^{-1}, \\
& L_{k}=-2 i y^{2} \frac{\partial}{\partial \bar{z}}=-i y^{2}\left(\frac{\partial}{\partial x}+i \frac{\partial}{\partial y}\right) .
\end{aligned}
$$

With respect to the Petersson slash operator (see (6.2)), these operators satisfy the intertwining properties

$$
\begin{aligned}
R_{k}\left(\left.f\right|_{k} \gamma\right) & =\left.\left(R_{k} f\right)\right|_{k+2} \gamma \\
L_{k}\left(\left.f\right|_{k} \gamma\right) & =\left.\left(L_{k} f\right)\right|_{k-2} \gamma
\end{aligned}
$$

for any $\gamma \in \mathrm{SL}_{2}(\mathbb{R})$. The Laplacian $\Delta_{k}$ can be expressed in terms of $R_{k}$ and $L_{k}$ by

$$
-\Delta_{k}=L_{k+2} R_{k}+k=R_{k-2} L_{k} .
$$

If $f$ is an eigenfunction of $\Delta_{k}$ satisfying $\Delta_{k} f=\lambda f$, then

$$
\begin{aligned}
\Delta_{k+2} R_{k} f & =(\lambda+k) R_{k} f, \\
\Delta_{k-2} L_{k} f & =(\lambda-k+2) L_{k} f .
\end{aligned}
$$

For any positive integer $n$ we put

$$
R_{k}^{n}:=R_{k+2(n-1)} \circ \cdots \circ R_{k+2} \circ R_{k} .
$$

We also let $R_{k}^{0}$ be the identity. The differential operator

$$
D:=\frac{1}{2 \pi i} \frac{d}{d z}=q \frac{d}{d q} .
$$

satisfies the following relation

$$
R_{k}=-4 \pi D+k / y .
$$

The next lemma is often referred to as Bol's identity.

Lemma 2.1. Assuming the notation and hypotheses above, we have

$$
D^{k-1}=\frac{1}{(-4 \pi)^{k-1}} R_{2-k}^{k-1}
$$

Proof. This is a special case of the identity (4.15) in [12].

\section{Proof of Theorem 1.1}

By Lemma 2.1, we see that $D^{k-1}$ defines a linear map from

$$
D^{k-1}: M_{2-k}^{!}\left(\Gamma_{0}(N), \chi\right) \longrightarrow M_{k}^{!}\left(\Gamma_{0}(N), \chi\right) .
$$

Theorem 1.1 asserts that this map may be extended to harmonic weak Maass forms. Moreover, the theorem provides a simple description of the images. 
Proof of Theorem 1.1. Suppose that $k \geq 2$, and that $f \in H_{2-k}\left(\Gamma_{0}(N), \chi\right)$. In view of Lemma 2.1, it is clear that $D^{k-1} f$ has the transformation behavior of a modular form of weight $k$.

We now show that $L_{k} D^{k-1} f=0$. This implies that $D^{k-1} f$ is holomorphic on $\mathbb{H}$. By Lemma 2.1, it suffices to show that $L_{k} R_{2-k}^{k-1} f=0$. Since $\Delta_{2-k} f=0$, it follows from (2.3) by induction that

$$
\Delta_{k-2} R_{2-k}^{k-2} f=(2-k) R_{2-k}^{k-2} f .
$$

Using (2.2), we obtain

$$
L_{k} R_{2-k}^{k-1} f=\left(L_{k} R_{k-2}\right) R_{2-k}^{k-2} f=\left(-\Delta_{k-2}-(k-2)\right) R_{2-k}^{k-2} f=0 .
$$

Finally, the growth behavior of $f$ at the cusps implies that $D^{k-1} f$ is meromorphic at the cusps. Therefore, $D^{k-1}$ indeed extends to $H_{2-k}\left(\Gamma_{0}(N), \chi\right)$.

To complete the proof, we compute the Fourier expansion of $D^{k-1} f$. Assuming the notation in (1.2), a straightforward calculation gives

$$
R_{2-k}^{k-2} f(z)=\sum_{n \gg-\infty} c_{f}^{+}(n) \Gamma(k-1,4 \pi n y)(-y)^{2-k} e^{2 \pi i n \bar{z}}+(k-2) !^{2} \sum_{n<0} c_{f}^{-}(n)(-y)^{2-k} e^{2 \pi i n \bar{z}} .
$$

Moreover, $R_{2-k}^{k-1} f$ has the Fourier expansion

$$
R_{2-k}^{k-1} f(z)=\sum_{n \gg-\infty} c_{f}^{+}(n)(-4 \pi n)^{k-1} q^{n}
$$

In particular, we have

$$
D^{k-1} f=D^{k-1} f^{+}=\sum_{n \gg-\infty} c_{f}^{+}(n) n^{k-1} q^{n} .
$$

The first two formulas follow from the Fourier expansion of $f$ and the differential equations $\Delta_{k-2} R_{2-k}^{k-2} f=(2-k) R_{2-k}^{k-2} f$ and $\Delta_{k} R_{2-k}^{k-1} f=0$. The third formula is a consequence of the second and Lemma 2.1.

Remark 7 . Note that $g:=y^{k-2} \overline{R_{2-k}^{k-2} f}$ is a harmonic weak Maass form of weight $2-k$ in the (slightly more general) sense of Section 3 of [7]. Moreover, $\xi_{2-k} g=y^{-k} \overline{L_{2-k} g}=R_{2-k}^{k-1} f$. This can also be used to compute the Fourier expansions in the proof of Theorem 1.1.

\section{The Regularized inner product and the Proof of Theorem 1.2}

Here we recall the regularized inner product, and we prove Theorem 1.2. We consider slightly more general situations, with earlier definitions modified in the obvious way.

Let $k$ be an integer, and let $\Gamma$ be a subgroup of finite index of $\Gamma(1)=\mathrm{SL}_{2}(\mathbb{Z})$. We define a regularized inner product of $g \in M_{k}(\Gamma)$ and $h \in M_{k}^{!}(\Gamma)$ as follows. For $T>0$ we denote by $\mathcal{F}_{T}(\Gamma(1))$ the truncated fundamental domain

$$
\mathcal{F}_{T}(\Gamma(1))=\{z \in \mathbb{H}:|x| \leq 1 / 2,|z| \geq 1, \text { and } y \leq T\}
$$


for $\Gamma(1)$. Moreover, we define the truncated fundamental domain for $\Gamma$ by

$$
\mathcal{F}_{T}(\Gamma)=\bigcup_{\gamma \in \Gamma \backslash \Gamma(1)} \gamma \mathcal{F}_{T}(\Gamma(1))
$$

Following [1], we define the regularized inner product $(g, h)^{r e g}$ as the constant term in the Laurent expansion at $s=0$ of the meromorphic continuation in $s$ of the function

$$
\frac{1}{[\Gamma(1): \Gamma]} \lim _{T \rightarrow \infty} \int_{\mathcal{F}_{T}(\Gamma)} g(z) \overline{h(z)} y^{k-s} \frac{d x d y}{y^{2}} .
$$

Using the same argument as in Section 6 of [1], it can be shown that $(g, h)^{\text {reg }}$ exists for any $g \in M_{k}(\Gamma)$ and $h \in M_{k}^{!}(\Gamma)$. (It also exists for $g \in M_{k}(\Gamma)$ and $h \in H_{k}(\Gamma)$. But note that it does not exist in general if $g$ and $h$ are both weakly holomorphic with honest poles at the cusps.) It is clear, for cusp forms $g$ and $h$, that the regularized inner product reduces to the classical Petersson inner product $(g, h)$.

Remark 8. If $h \in M_{k}^{!}(\Gamma)$ has vanishing constant term at every cusp of $\Gamma$, then

$$
(g, h)^{r e g}=\frac{1}{[\Gamma(1): \Gamma]} \lim _{T \rightarrow \infty} \int_{\mathcal{F}_{T}(\Gamma)} g(z) \overline{h(z)} y^{k} \frac{d x d y}{y^{2}} .
$$

For the rest of this section we assume that $k \geq 2$.

Theorem 4.1. If $g \in M_{k}(\Gamma)$ and $f \in H_{2-k}(\Gamma)$, then

$$
\left(g, R_{2-k}^{k-1} f\right)^{r e g}=\frac{(-1)^{k}}{[\Gamma(1): \Gamma]} \sum_{\kappa \in \Gamma \backslash P^{1}(\mathbb{Q})} w_{\kappa} \cdot c_{g}(0, \kappa) \overline{c_{f}^{+}(0, \kappa)},
$$

where $c_{g}(0, \kappa)$ (resp. $c_{f}^{+}(0, \kappa)$ ) denotes the constant term of the Fourier expansion of $g$ (resp. f) at the cusp $\kappa \in P^{1}(\mathbb{Q})$, and $w_{\kappa}$ is the width of the cusp $\kappa$.

Proof. For simplicity, we carry out the proof only in the special case $\Gamma=\Gamma(1)$. The general case is completely analogous. We put $H:=y^{k-2} \overline{R_{2-k}^{k-2} f}$. Then $h:=R_{2-k}^{k-1} f=y^{-k} \overline{L_{2-k} H}$. Since the constant terms at all cusps of $h$ vanish, we have

$$
\begin{aligned}
\left(g, R_{2-k}^{k-1} f\right)^{r e g} & =\lim _{T \rightarrow \infty} \int_{\mathcal{F}_{T}(\Gamma)} g(z) \overline{h(z)} y^{k} \frac{d x d y}{y^{2}} \\
& =\lim _{T \rightarrow \infty} \int_{\mathcal{F}_{T}(\Gamma)} g(z)\left(L_{2-k} H\right) \frac{d x d y}{y^{2}} \\
& =\lim _{T \rightarrow \infty} \int_{\mathcal{F}_{T}(\Gamma)} g(z)\left(\frac{\partial}{\partial \bar{z}} H\right) d z d \bar{z} \\
& =-\lim _{T \rightarrow \infty} \int_{\mathcal{F}_{T}(\Gamma)}(\bar{\partial} H) \wedge g(z) d z
\end{aligned}
$$


Using the holomorphy of $g$, we obtain, by Stokes' theorem, the expression

$$
\begin{aligned}
\left(g, R_{2-k}^{k-1} f\right)^{r e g} & =-\lim _{T \rightarrow \infty} \int_{\mathcal{F}_{T}(\Gamma)} d(H(z) g(z) d z) \\
& =-\lim _{T \rightarrow \infty} \int_{\partial \mathcal{F}_{T}(\Gamma)} H(z) g(z) d z \\
& =\lim _{T \rightarrow \infty} \int_{x=-1 / 2}^{1 / 2} H(x+i T) g(x+i T) d x .
\end{aligned}
$$

The integral over $x$ gives the constant term in the Fourier expansion of $H(x+i T) g(x+i T)$. It can be computed using the Fourier expansion

$$
H(z)=(-1)^{k} \sum_{n \gg-\infty} \overline{c_{f}^{+}(n)} \Gamma(k-1,4 \pi n y) e^{-2 \pi i n z}+(-1)^{k}(k-2) !^{2} \sum_{n<0} \overline{c_{f}^{-}(n)} e^{-2 \pi i n z}
$$

of $H$ (see the proof of Theorem 1.1) and the Fourier expansion of $g$. It turns out that under the limit $T \rightarrow \infty$ only the contribution $(-1)^{k} c_{g}(0) \overline{c_{f}^{+}(0)}$ coming from the product of the individual constant terms survives. This concludes the proof.

Corollary 4.2. If $g \in S_{k}(\Gamma)$, then $\left(g, R_{2-k}^{k-1} f\right)^{r e g}=0$.

Proof. This is a direct consequence of Theorem 4.1.

The next corollary implies Theorem 1.2.

Corollary 4.3. The image of the map $D^{k-1}: H_{2-k}(\Gamma) \rightarrow M_{k}^{!}(\Gamma)$ is given by those $h \in$ $M_{k}^{!}(\Gamma)$ which are orthogonal to cusp forms and whose constant term at any cusp of $\Gamma$ vanishes.

Proof. If $f \in H_{2-k}(\Gamma)$, it follows from Theorem 4.1 and Theorem 1.1 that $D^{k-1} f$ satisfies the stated conditions.

Conversely, assume that $h \in M_{k}^{!}(\Gamma)$ is orthogonal to cusp forms and has vanishing constant term at any cusp of $\Gamma$. According to Lemma 3.11 of [7], we may chose $f \in$ $H_{2-k}(\Gamma)$ such that the principal parts of $D^{k-1} f$ and $h$ at the cusps agree up to the constant terms. Since the constant terms of $h$ and $D^{k-1} f$ vanish, they trivially agree as well. Consequently,

$$
h-D^{k-1} f \in S_{k}(\Gamma) .
$$

In view of Theorem 4.1 and the hypothesis on $h$, we find that $h-D^{k-1} f$ is orthogonal to cusp forms. Hence it vanishes identically.

Remark 9. It suffices to specialize $\Gamma=\Gamma_{1}(N)$ in the previous Corollary in order to derive Theorem 1.2 .

\section{Proof of Theorems 1.3, 1.4, And 1.5}

Here we prove Theorem 1.3 by combining facts about $\xi_{2-k}$, with Hecke theory and the theory of complex multiplication. We first begin with an important proposition. 
Proposition 5.1. Let $g=\sum_{n=1}^{\infty} b(n) q^{n} \in S_{k}\left(\Gamma_{0}(N), \bar{\chi}\right)$ be a normalized newform with integer weight $k \geq 2$, and let $F_{g}$ be the number field obtained by adjoining the coefficients of $g$ to $\mathbb{Q}$. Then there is a harmonic weak Maass form $f \in H_{2-k}\left(\Gamma_{0}(N), \chi\right)$ which satisfies:

(i) The principal part of $f$ at the cusp $\infty$ belongs to $F_{g}\left[q^{-1}\right]$.

(ii) The principal parts of $f$ at the other cusps of $\Gamma_{0}(N)$ are constant.

(iii) We have that $\xi_{2-k}(f)=\|g\|^{-2} g$.

Proof. Let $H_{2-k, \infty}\left(\Gamma_{0}(N), \chi\right)$ be the subspace of those $f \in H_{2-k}\left(\Gamma_{0}(N), \chi\right)$ whose principal parts at the cusps other than $\infty$ are constant. Note that

$$
H_{2-k}\left(\Gamma_{0}(N), \chi\right)=H_{2-k, \infty}\left(\Gamma_{0}(N), \chi\right)+M_{2-k}^{!}\left(\Gamma_{0}(N), \chi\right) .
$$

Arguing as in Section 3 of [7], the restriction of $\xi_{2-k}$ to $H_{2-k, \infty}\left(\Gamma_{0}(N), \chi\right)$ defines a surjective map to $S_{k}\left(\Gamma_{0}(N), \bar{\chi}\right)$. One now argues as in the proof of Lemma 7.3 of [8] using the pairing $\{g, f\}=\left(g, \xi_{2-k}(f)\right)$, where $f \in H_{2-k, \infty}\left(\Gamma_{0}(N), \chi\right)$ and $g \in S_{k}\left(\Gamma_{0}(N), \bar{\chi}\right)$.

Remark 10. The harmonic weak Maass form $f$ satisfying (i)-(iii) above is unique up to the addition of a weakly holomorphic form in $M_{2-k}^{!}\left(\Gamma_{0}(N), \chi\right)$ with coefficients in $F_{g}$ and a pole possibly at infinity and constant principal part at all other cusps.

For completeness, here we briefly recall the notion of a newform with complex multiplication (for example, see Chapter 12 of [11] or Section 1.2 of [13]). Let $D<0$ be the fundamental discriminant of an imaginary quadratic field $K=\mathbb{Q}(\sqrt{D})$. Let $O_{K}$ be the ring of integers of $K$, and let $\chi_{K}:=\left(\frac{D}{\bullet}\right)$ be the usual Kronecker character associated to $K$. Let $k \geq 2$, and let $c$ be a Hecke character of $K$ with exponent $k-1$ and conductor $\mathfrak{f}_{c}$, a non-zero ideal of $O_{K}$. By definition, this means that

$$
c: I\left(\mathfrak{f}_{c}\right) \longrightarrow \mathbb{C}^{\times}
$$

is a homomorphism, where $I\left(\mathfrak{f}_{c}\right)$ denotes the group of fractional ideals of $K$ prime to $\mathfrak{f}_{c}$. In particular, this means that

$$
c\left(\alpha O_{K}\right)=\alpha^{k-1}
$$

for $\alpha \in K^{\times}$for which $\alpha \equiv 1 \bmod ^{\times} \mathfrak{f}_{c}$. To $c$ we naturally associate a Dirichlet character $\omega_{c}$ defined, for every integer $n$ coprime to $\mathfrak{f}_{c}$, by

$$
\omega_{c}(n):=\frac{c\left(n O_{K}\right)}{n^{k-1}} .
$$

Given this data, we let

$$
\Phi_{K, c}(z):=\sum_{\mathfrak{a}} c(\mathfrak{a}) q^{N(a)}
$$

where $\mathfrak{a}$ varies over the ideals of $O_{K}$ prime to $\mathfrak{f}_{c}$, and where $N(\mathfrak{a})$ is the usual ideal norm. It is well known that $\Phi_{K, c}(z) \in S_{k}\left(\Gamma_{0}\left(|D| \cdot N\left(\mathfrak{f}_{c}\right)\right), \chi_{K} \cdot \omega_{c}\right)$ is a normalized newform. These are newforms with complex multiplication. By construction, if we let

$$
\Phi_{K, c}(z)=\sum_{n=1}^{\infty} b(n) q^{n},
$$


then

$$
b(n)=0 \quad \text { whenever } \chi_{K}(n)=-1 .
$$

This follows since every prime $p$ for which $\chi_{K}(p)=-1$ is inert.

Proof of Theorem 1.3. Suppose that $f$ is good for a CM form $g=\sum_{n=1}^{\infty} b(n) q^{n}$, and let $D=D_{g}$ be the fundamental discriminant of the associated imaginary quadratic field $K=\mathbb{Q}(\sqrt{D})$. By (1.3) (correcting a typographical error in Lemma 3.1 of [7]), we then have that

$$
\xi_{2-k}(f)=\|g\|^{-2} g=-(4 \pi)^{k-1} \sum_{n=1}^{\infty} \overline{c_{f}^{-}(-n)} n^{k-1} q^{n} .
$$

Since $g$ has complex multiplication, (5.2) implies that $c_{f}^{-}(n)=0$ when $\chi_{K}(-n)=-1$. Because $D<0$, this means that

$$
c_{f}^{-}(n)=0 \text { when } \chi_{K}(n)=1 .
$$

Let $M=N D$. We write $\chi_{0}$ for the trivial character modulo $|D|$. Since $D \mid N$, a standard argument shows that the sum of character twists

$$
u:=f \otimes \chi_{0}+f \otimes \chi_{K}
$$

is in $H_{2-k}\left(\Gamma_{0}(M), \chi\right)$. The Fourier expansion of $u=u^{+}+u^{-}$is given by

$$
\begin{aligned}
& u^{+}(z)=2 \sum_{\substack{n \gg-\infty \\
\chi_{K}(n)=1}} c_{f}^{+}(n) q^{n}, \\
& u^{-}(z)=2 \sum_{\substack{n<0 \\
\chi_{K}(n)=1}} c_{f}^{-}(n) \Gamma(k-1,4 \pi|n| y) q^{n} .
\end{aligned}
$$

Consequently, by (5.3), the non-holomorphic part $u^{-}$vanishes, and $u$ is actually weakly holomorphic.

We now claim that for any integer $b, f(z+b / D)$ has principal parts at all cusps in $F_{g}\left(\zeta_{M}\right)\left[q^{-1}\right]$. To see this, we let $\gamma \in \Gamma(1)$ and consider the cusp $\gamma \infty$. There exists a $\tilde{\gamma} \in \Gamma(1)$ and $\alpha, \beta, \delta \in \mathbb{Z}$ such that

$$
\left(\begin{array}{cc}
D & b \\
0 & D
\end{array}\right) \gamma=\tilde{\gamma}\left(\begin{array}{ll}
\alpha & \beta \\
0 & \delta
\end{array}\right)
$$

Hence, the Fourier expansion of $f(z+b / D)$ at the cusp $\gamma \infty$ is given by

$$
f|\tilde{\gamma}|\left(\begin{array}{ll}
\alpha & \beta \\
0 & \delta
\end{array}\right) \text {. }
$$

By the assumption of $f$, it is holomorphic at the cusp $\infty$, unless $\tilde{\gamma} \in \Gamma_{0}(N)$, in which case it is equal to

$$
f \mid\left(\begin{array}{ll}
\alpha & \beta \\
0 & \delta
\end{array}\right)
$$


Since $\delta\left|D^{2}\right| M$, the principal part at $\infty$ of this modular form is contained in $F_{g}\left(\zeta_{M}\right)\left[q^{-1}\right]$, proving the claim. This implies that the twists $f \otimes \chi_{0}, f \otimes \chi_{D}$, have principal parts at all cusps in $F_{g}\left(\zeta_{M}\right)\left[q^{-1}\right]$. Therefore, the same is true for $u$.

Now we recall the fact that the action of $\operatorname{Aut}\left(\mathbb{C} / \mathbb{Q}\left(\zeta_{N}\right)\right)$ commutes with the action of $S L_{2}(\mathbb{Z})$ on modular functions for $\Gamma(N)$ (for example, see Theorem 6.6 in Chapter 6.2 and the diagram before Remark 6.7 in Shimura's book [19]). Using the action of $\operatorname{Aut}\left(\mathbb{C} / F_{g}\left(\zeta_{M}\right)\right)$ on weakly holomorphic modular forms, we see that $u^{\sigma}$ has the same properties for any $\sigma \in \operatorname{Aut}\left(\mathbb{C} / F_{g}\left(\zeta_{M}\right)\right)$. Moreover, $u^{\sigma}$ has the same principal parts as $u$ at all cusps. Hence the difference $u-u^{\sigma}$ is a holomorphic modular form which vanishes at the cusp $\infty$. Since $2-k \leq 0$, this implies that $u=u^{\sigma}$. Consequently, $u$ is defined over $F_{g}\left(\zeta_{M}\right)$. So for all $n \in \mathbb{Z}$ with $\chi_{K}(n)=1$, we have that $c_{f}^{+}(n) \in F_{g}\left(\zeta_{M}\right)$. In particular, $c_{f}^{+}(1) \in F_{g}\left(\zeta_{M}\right)$.

We now use the Hecke action on $f$ and $g$. Let $T(m)$ be the $m$-th Hecke operator for $\Gamma_{0}(N)$. Using the same argument as in Lemma 7.4 of [8], we have that

$$
\left.f\right|_{2-k} T(m)=m^{1-k} b(m) f+f^{\prime},
$$

where $f^{\prime} \in M_{2-k}^{!}\left(\Gamma_{0}(N), \chi\right)$ is a weakly holomorphic form with coefficients in $F_{g}$. In view of the formula for the action of the Hecke operators on the Fourier expansion, we obtain for any prime $p$ that

$$
c_{f}^{+}(p n)+\chi(p) p^{1-k} c_{f}^{+}(n / p)=p^{1-k} b(p) c_{f}^{+}(n)+c_{f^{\prime}}^{+}(n),
$$

where $c_{f^{\prime}}^{+}(n) \in F_{g}$. Hence an inductive argument shows that all coefficients $c_{f}^{+}(n)$ are contained in the extension $F_{g}\left(c_{f}^{+}(1)\right)$. This concludes the proof of the theorem since we have already established that $c_{f}^{+}(1)$ is in $F_{g}\left(\zeta_{M}\right)$.

The proof of Theorem 1.4 is similar to the proof of Theorem [1.3, and so we give only a sketch of the set-up.

Sketch proof of Theorem 1.4. If $p \nmid N$ is a prime, then for every positive integer $m$ we have that

$$
c_{g}(p) c_{g}\left(p^{m}\right)=c_{g}\left(p^{m+1}\right)+\overline{\chi(p)} p^{k-1} c_{g}\left(p^{m-1}\right) .
$$

Therefore, if $p \nmid N$ is a prime for which $c_{g}(p)=0$, then we have that

$$
c_{g}\left(p^{m+1}\right)=-\overline{\chi(p)} p^{k-1} c_{g}\left(p^{m-1}\right),
$$

which in turn implies that

$$
c_{g}\left(p^{m}\right)= \begin{cases}\left(-\overline{\chi(p)} p^{k-1}\right)^{\frac{m}{2}} & \text { if } m \text { is even } \\ 0 & \text { otherwise. }\end{cases}
$$

Therefore, by arguing with the usual $U(p), V(p), U\left(p^{2}\right)$ and $V\left(p^{2}\right)$ operators, we can obtain a harmonic weak Maass form whose Fourier coefficients are supported on terms whose exponents $n$ have the property that $p$ exactly divides $n$. By the multiplicativity of the Fourier coefficients of newforms, it then follows by the observation above that the nonholomorphic part of this form is identically zero. In other words, this particular harmonic 
weak Maass form is a weakly holomorphic modular form with suitable principal parts at cusps. The proof now follows mutatis mutandis as in the proof of Theorem 1.3.

5.1. Proof of Theorem 1.5. Theorem 1.5 follows easily from the seminal work of Serre [17, 18] on $p$-adic modular forms. We now apply his works to prove the theorem.

By Theorem 1.1, we have that

$$
\sum_{n \gg-\infty} c_{f}^{+}(n) n^{k-1} q^{n}
$$

is a weight $k$ weakly holomorphic modular form on $\Gamma_{0}\left(p^{t}\right)$ with rational coefficients. By standard facts involving the $U(p)$-operator

$$
\left(\sum a(n) q^{n}\right) \mid U(p):=\sum a(n p) q^{n}
$$

the integer $a$ has the property that

$$
f^{*}(z):=\sum_{n=0}^{\infty} c_{f}^{+}\left(p^{a} n\right) n^{k-1} q^{n}
$$

is a weight $k$ weakly holomorphic modular form on $\Gamma_{0}\left(p^{t^{*}}\right)$, where $t^{*}=1$ if $t=0$, and is $t$ otherwise. This modular form has trivial principal part at the cusp infinity. Therefore, we may apply a theorem of Serre (see Th. 5.4 of [18]), and the conclusion is that $f^{*}(z)$ is a $p$-adic modular form on $\mathrm{SL}_{2}(\mathbb{Z})$ of weight $k$. This proves (1).

Claim (2) follows from the definition of a $p$-adic modular form. Indeed, $p$-adic modular forms are $p$-adic limits of the Fourier expansions of classical holomorphic modular forms, and these forms, by a theorem of Serre, have the property that almost all of their coefficients are multiples of any fixed power of $p$ (see Th. 4.7 of [18]). This implies (2).

Claim (3) is a consequence of the fact that the $U(p)$-operator acts locally nilpotently on certain $p$-adic modular forms. In this situation, Serre proved that the constant term of a $p$-adic modular form is a limit, in the $p$-adic sense, of certain Fourier coefficients. This result (see Th. 7 and the following remark in [17]) implies (3).

\section{Poincaré series and Theorems 1.1, 1.2, and 1.3}

Here we consider natural examples of the results of both Theorems [1.1, 1.2 and [1.3, Our results depend on the explicit Fourier expansions of two classes of Poincaré series (for example, see [4, 6, 11]).

6.1. Definitions and Fourier expansions. For $A=\left(\begin{array}{ll}a & b \\ c & d\end{array}\right) \in \mathrm{SL}_{2}(\mathbb{Z})$, define $j(A, z)$ by

$$
j(A, z):=(c z+d) .
$$

As usual, for such $A$ and functions $f: \mathbb{H} \rightarrow \mathbb{C}$, we let

$$
\left(\left.f\right|_{k} A\right)(z):=j(A, z)^{-k} f(A z) .
$$

Let $m$ be an integer, and let $\varphi_{m}: \mathbb{R}^{+} \rightarrow \mathbb{C}$ be a function which satisfies $\varphi_{m}(y)=O\left(y^{\alpha}\right)$, as $y \rightarrow 0$, for some $\alpha \in \mathbb{R}$. If $e(\alpha):=e^{2 \pi i \alpha}$ as before, then let

$$
\varphi_{m}^{*}(z):=\varphi_{m}(y) e(m x) .
$$


Such functions are fixed by the translations $\Gamma_{\infty}:=\left\{ \pm\left(\begin{array}{ll}1 & n \\ 0 & 1\end{array}\right): n \in \mathbb{Z}\right\}$.

Given this data, for integers $N \geq 1$, we define the generic Poincaré series

$$
\mathbb{P}\left(m, k, \varphi_{m}, N ; z\right):=\sum_{A \in \Gamma_{\infty} \backslash \Gamma_{0}(N)}\left(\left.\varphi_{m}^{*}\right|_{k} A\right)(z) .
$$

We shall be interested in two families of such series.

The first family is classical. We let

$$
P(m, k, N ; z)=q^{m}+\sum_{n=1}^{\infty} a(m, k, N ; n) q^{n}:=\mathbb{P}(m, k, e(i m y), N ; z) .
$$

These series are modular, and their Fourier expansions are given in terms of the $I$-Bessel and $J$-Bessel functions, and the Kloosterman sums

$$
K(m, n, c):=\sum_{v(c) \times} e\left(\frac{m \bar{v}+n v}{c}\right) .
$$

Here $v$ runs through the primitive residue classes modulo $c$, and $v \bar{v} \equiv 1(\bmod c)$. The following is well known (for example, see [11, 15]).

Proposition 6.1. If $k \in 2 \mathbb{N}$, and $m, N \geq 1$, then the following are true.

1) We have that $P(m, k, N ; z) \in S_{k}\left(\Gamma_{0}(N)\right)$, and for positive integers $n$ we have

$$
a(m, k, N ; n)=2 \pi(-1)^{\frac{k}{2}}\left(\frac{n}{m}\right)^{\frac{k-1}{2}} \cdot \sum_{\substack{c>0 \\ c \equiv 0}} \frac{K(m, n, c)}{c} \cdot J_{k-1}\left(\frac{4 \pi \sqrt{m n}}{c}\right) .
$$

2) We have that $P(-m, k, N ; z) \in M_{k}^{!}\left(\Gamma_{0}(N)\right)$, and for positive integers $n$ we have

$$
a(-m, k, N ; n)=2 \pi(-1)^{\frac{k}{2}}\left(\frac{n}{m}\right)^{\frac{k-1}{2}} . \sum_{\substack{c>0 \\ c \equiv 0}} \frac{K(-m, n, c)}{c} \cdot I_{k-1}\left(\frac{4 \pi \sqrt{|m n|}}{c}\right) .
$$

Now we recall the second family of Poincaré series, the Maass-Poincaré series of Hejhal (see [10]). Let $M_{\nu, \mu}(z)$ be the usual $M$-Whittaker function. For complex $s$, let

$$
\mathcal{M}_{s}(y):=|y|^{-\frac{k}{2}} M_{\frac{k}{2} \operatorname{sgn}(y), s-\frac{1}{2}}(|y|)
$$

and for $m \geq 1$ let $\varphi_{-m}(z):=\mathcal{M}_{1-\frac{k}{2}}(-4 \pi m y)$. For $k \in 2 \mathbb{N}$ and integers $N \geq 1$, we let

$$
Q(-m, k, N ; z):=\frac{1}{(k-1) !} \cdot \mathbb{P}\left(-m, 2-k, \varphi_{-m}, N ; z\right) .
$$

To determine the Fourier expansions of these series, we also require the incomplete Gamma-function $\Gamma(a, x)$. We have the following proposition (for example, see [4, 6, 10]).

Proposition 6.2. If $k \in 2 \mathbb{N}$, and $m, N \geq 1$, then $Q(-m, k, N ; z) \in H_{2-k}\left(\Gamma_{0}(N)\right)$, and has a Fourier expansion of the form

$$
Q(-m, k, N ; z)=Q^{+}(-m, k, N ; z)+Q^{-}(-m, k, N ; z),
$$


where

$$
Q^{-}(-m, k, N ; z)=-\frac{\Gamma(k-1,4 \pi m y)}{(k-2) !} q^{-m}+\sum_{n<0} b(-m, k, N ; n) \cdot \Gamma(k-1,4 \pi|n| y) q^{n}
$$

and where for negative integers $n$ we have

$$
b(-m, k, N ; n)=-\frac{2 \pi(-1)^{\frac{k}{2}}}{(k-2) !} \cdot\left|\frac{m}{n}\right|^{\frac{k-1}{2}} \sum_{\substack{c>0 \\ c \equiv 0}} \frac{K(-m, n, c)}{c} \cdot J_{k-1}\left(\frac{4 \pi \sqrt{|m n|}}{c}\right),
$$

and

$$
Q^{+}(-m, k, N ; z)=q^{-m}+\sum_{n=0}^{\infty} b(-m, k, N ; n) q^{n}
$$

where

$$
b(-m, k, N ; 0)=-\frac{2^{k} \pi^{k}(-1)^{\frac{k}{2}} m^{k-1}}{(k-1) !} \cdot \sum_{c \equiv 0 \underset{c>0}{(\bmod N)}} \frac{K(-m, 0, c)}{c^{k}},
$$

and where for positive integers $n$ we have

$$
b(-m, k, N ; n)=-2 \pi(-1)^{\frac{k}{2}} . \sum_{\substack{c>0 \\(\bmod N)}}\left(\frac{m}{n}\right)^{\frac{k-1}{2}} \frac{K(-m, n, c)}{c} \cdot I_{k-1}\left(\frac{4 \pi \sqrt{|m n|}}{c}\right) .
$$

Remark 11. Obviously, we have that $Q^{+}(-m, k, N ; z)\left(\operatorname{resp} . \quad Q^{-}(-m, k, N ; z)\right)$ is the holomorphic part (resp. non-holomorphic part) of the weak Maass form $Q(-m, k, N ; z)$.

Remark 12. Propositions 6.1 and 6.2 are well known for $2<k \in 2 \mathbb{N}$. That they hold for $k=2$ follows by arguing by analytic continuation in $k$ with Fourier expansions.

6.2. Poincaré series in the context of Theorems $\mathbf{1 . 1}$ and $\mathbf{1 . 2}$. If $k \geq 2$ is even, and $m, N \geq 1$, then Theorem 1.1, and Propositions 6.1 and 6.2, imply that

$$
\begin{aligned}
& D^{k-1} Q(-m, k, N ; z)=D^{k-1} Q^{+}(-m, k, N ; z)= \\
& \quad=(-m)^{k-1} q^{-m}-2 \pi(-1)^{\frac{k}{2}} \cdot \sum_{n=1}^{\infty} \sum_{c \equiv 0}(m n)^{\frac{k-1}{2}} \frac{K(-m, n, c)}{c} \cdot I_{k-1}\left(\frac{4 \pi \sqrt{|m n|}}{c}\right) \\
& \quad=-m^{k-1} P(-m, k, N ; z) .
\end{aligned}
$$

In other words, we have

$$
D^{k-1} Q(-m, k, N ; z)=-m^{k-1} P(-m, k, N ; z) .
$$

By (1.3), we also have that

$$
\xi_{2-k}(Q(-m, k, N ; z))=\frac{(4 \pi)^{k-1} m^{k-1}}{(k-2) !} \cdot P(m, k, N ; z) .
$$


For one dimensional spaces $S_{k}\left(\Gamma_{0}(N)\right)$, say generated by a newform $g$, this last relation relates Maass-Poincaré series to newforms $g$. This follows from standard facts about Petersson inner products and Poincaré series (for example, see Chapter 3 of [1]]).

\section{A GOOD EXAMPLE}

Correspondence (6.8) can be useful for computing Fourier coefficients of holomorphic parts of certain harmonic weak Maass forms. Here we consider example (1.8) from the introduction. Using Proposition 6.2, we summed the first 150 terms to obtain

$$
\begin{aligned}
-D^{3} Q(-1,4,9 ; z) & =P(-1,4,9 ; z) \\
& \sim q^{-1}+1.9999 q^{2}-48.9999 q^{5}+47.9999 q^{8}+770.9999 q^{11}+\cdots .
\end{aligned}
$$

On the other hand, we have the weight 4 weakly holomorphic modular form

$$
m(z):=\left(\frac{\eta(z)^{3}}{\eta(9 z)^{3}}+3\right)^{2} \cdot \eta(3 z)^{8}=q^{-1}+2 q^{2}-49 q^{5}+48 q^{8}+771 q^{11}-\cdots,
$$

where $\eta(z):=q^{\frac{1}{24}} \prod_{n=1}^{\infty}\left(1-q^{n}\right)$ is Dedekind's eta-function. These two modular forms are equal, and so the coefficients are obviously rational (in fact, integral). To deduce this, one may use the "circle method" to get asymptotics for the coefficients of $m(z)$ (for example, see the detailed discussion in [16]). The circle method gives the same asymptotic expressions for the coefficients of $m(z)$ and $P(-1,4,9 ; z)$. This follows from the fact that they have the same principal parts at cusps. The circle method then shows that these approximations for the $n$th coefficients of $m(z)$ and $P(-1,4,9 ; z)$ agree up to a power of $n$. This follows from a standard calculation involving bounds for Kloosterman sums and the asymptotic properties of $I$-Bessel functions. Therefore, it follows that $P(-1,4,9 ; z)-m(z)$ is a holomorphic modular form. This form vanishes at all cusps, and so it must be a cusp form. The space $S_{4}\left(\Gamma_{0}(9)\right)$ is one dimensional, and is spanned by the CM form

$$
g(z)=\eta(3 z)^{8}=q-8 q^{4}+20 q^{7}-70 q^{13}+64 q^{16}+56 q^{19}+\cdots .
$$

The non-zero coefficients of this cusp form have exponents $n$ in the arithmetic progression $n \equiv 1(\bmod 3)$. However, since $K(-1, n, 9 c)=0$ for integers $n \equiv 1(\bmod 3)$, Proposition 6.1 (2) then implies that $P(-1,4,9 ; z)-m(z)$ is identically zero. Combining these facts, we find that the coefficients of $Q^{+}(-1,4,9 ; z)$ are rational, and its first few terms are

$$
Q^{+}(-1,4,9 ; z)=q^{-1}-\frac{1}{4} q^{2}+\frac{49}{125} q^{5}-\frac{48}{512} q^{8}-\frac{771}{1331} q^{11}+\cdots
$$

In the context of Theorem 1.3, the algebraicity of $Q^{+}(-1,4,9 ; z)$ follows from the fact that $Q(-1,4,9 ; z)$ is good for the CM form $g(z)$. To see this one observes that $\xi_{-2}(Q(-1,4,9 ; z))=\|g\|^{-2} g$. This follows from Petersson's theory, combined with the fact that $S_{4}\left(\Gamma_{0}(9)\right)$ is one dimensional, and is spanned by both $g(z)$ and $P(1,4,9 ; z)$.

Theorem [1.5 is also easily described in this example. We have that $a=1$ in Theorem 1.5, and so the $q$-series $f^{*}$ in Theorem 1.5 (1) is $-m(z) \mid U(3)$. Theorem 1.5 (2) then implies that almost every coefficient of $m(z) \mid U(3)$ is a multiple of any fixed power of 3 . 
Theorem 1.5 (3) follows trivially in this case since the coefficients of $m(z)$ for exponents which are powers of 3 obviously vanish.

\section{REFERENCES}

[1] R. Borcherds, Automorphic forms with singularities on Grassmannians, Invent. Math. 132 (1998), pages $491-562$.

[2] K. Bringmann and K. Ono, The $f(q)$ mock theta function conjecture and partition ranks, Invent. Math. 165 (2006), pages 243-266.

[3] K. Bringmann and K. Ono, Dyson's ranks and Maass forms, Ann. of Math., in press.

[4] K. Bringmann and K. Ono, Lifting cusp forms to Maass forms with an application to partitions, Proc. Natl. Acad. Sci., USA 104, No. 10 (2007), pages 3725-3731.

[5] K. Bringmann, K. Ono, and R. C. Rhoades, Eulerian series as modular forms, J. Amer. Math. Soc., in press.

[6] J. H. Bruinier, Borcherds products on $O(2, l)$ and Chern classes of Heegner divisors, Springer Lecture Notes in Mathematics 1780, Springer-Verlag (2002).

[7] J. H. Bruinier and J. Funke, On two geometric theta lifts, Duke Math. J. 125 (2004), pages $45-90$.

[8] J. H. Bruinier and K. Ono, Heegner divisors, $L$-functions, and harmonic weak Maass forms, preprint.

[9] D. Bump, Automorphic forms and representations, Cambridge Univ. Press, Cambridge, 1997.

[10] D. A. Hejhal, The Selberg trace formula for $P S L(2, \mathbb{R})$, Lect. Notes. in Math. 1001, SpringerVerlag, Berlin, 1983.

[11] H. Iwaniec, Topics in the classical theory of automorphic forms, Grad. Studies in Math. 17, Amer. Math. Soc., Providence, R.I., 1997.

[12] J. Lewis and D. Zagier, Period functions for Maass wave forms. I., Ann. Math. 153 (2001), pages 191-258.

[13] K. Ono, The web of modularity: arithmetic of the coefficients of modular forms and $q$-series, Conference Board of the Mathematical Sciences 102, Amer. Math. Soc. (2004).

[14] K. Ono, A mock theta function for the Delta-function, Proceedings of the 2007 Integers Conference, to appear.

[15] H. Petersson, Über die Entwicklungskoeffizienten der automorphen Formen, Acta Math. 58 (1932), pages 169-215.

[16] H. Rademacher, Topics in analytic number theory, Springer-Verlag, New York, 1973.

[17] J.-P. Serre, Formes modulaires et fonctions zêta $p$-adiques, Springer Lect. Notes in Math. 350, Springer-Verlag, Berlin, (1973), pages 191-268.

[18] J.-P. Serre, Divisibilité de certaines fonctions arithmétiques, L'Enseign. Math. 22 (1976), pages $227-260$.

[19] G. Shimura, Introduction to the arithmetic theory of automorphic functions, Princeton Univ. Press, Princeton, NJ, 1994.

[20] D. Zagier, Ramanujan's mock theta functions and their applications [d'après Zwegers and Bringmann-Ono], Sém. Bourbaki (2007), in press.

[21] S. P. Zwegers, Mock theta functions, Ph.D. Thesis, Universiteit Utrecht, 2002.

Fachbereich Mathematik, Technische Universität Darmstadt, Schlossgartenstrasse 7, D-64289 Darmstadt, Germany

E-mail address: bruinier@mathematik.tu-darmstadt.de 
Department of Mathematics, University of Wisconsin, Madison, Wisconsin 53706 USA E-mail address: ono@math.wisc.edu

E-mail address: rhoades@math.wisc.edu 